PAPER

\title{
Temporal evolution of water diffusion parameters is different in grey and white matter in human ischaemic stroke
}

\author{
S Muñoz Maniega, M E Bastin, P A Armitage, A J Farrall, T K Carpenter, P J Hand, V Cvoro, \\ C S Rivers, J M Wardlaw
}

J Neurol Neurosurg Psychiatry 2004;75:1714-1718. doi: 10.1136/jnnp.2003.033852

See end of article for authors' affiliations

Correspondence to: DrM Bastin Medical and Radiological Sciences (Medical Physics)

University of Edinburgh, Western General Hospital, Crewe Road, Edinburgh, EH4 2XU; meb@skull.dcn. ed.ac.uk

Received 5December 2003 In revised form

16 March 2004

Accepted 22 March 2004

\begin{abstract}
Objectives: Our purpose was to investigate whether differences exist in the values and temporal evolution of mean diffusivity $(<D>)$ and fractional anisotropy (FA) of grey and white matter after human ischaemic stroke.

Methods: Thirty two patients with lesions affecting both grey and white matter underwent serial diffusion tensor magnetic resonance imaging (DT-MRI) within 24 hours, and at 4-7 days, 10-14 days, 1 month, and 3 months after stroke. Multiple small circular regions of interest (ROI) were placed in the grey and white matter within the lesion and in the contralateral hemisphere. Values of $\angle D>\{$ grey\}, $\angle D>\{$ white , $\mathrm{FA}\{$ grey\} and $\mathrm{FA}\{$ white $\}$ were measured in these $\mathrm{ROI}$ at each time point and the ratios of ischaemic to normal contralateral values $\left(\angle D>_{R}\right.$ and $\left.F A_{R}\right)$ calculated.

Results: $\angle D>$ and FA showed different patterns of evolution after stroke. After an initial decline, the rate of increase of $\langle D\rangle$ \{grey\} was faster than $\langle D>\{$ white $\}$ from 4-7 to 10-14 days. FA\{white $\}$ decreased more rapidly than FA\{grey\} during the first week, thereafter for both tissue types the FA decreased gradually. However, FA\{white $\}$ was still higher than FA\{grey\} at three months indicating that some organised axonal structure remained. This effect was more marked in some patients than in others. $\left\langle\mathrm{D}>_{\mathrm{R}}\{\right.$ grey $\}$ was significantly higher than $<D>_{R}\{$ white $\}$ within 24 hours and at 10-14 days $(p<0.05)$, and $F A_{R}\{$ white $\}$ was significantly more reduced than $F A_{R}\{g r e y\}$ at all time points $(p<0.001)$.

Conclusions: The values and temporal evolution of $\angle D>$ and FA are different for grey and white matter after human ischaemic stroke. The observation that there is patient-to-patient variability in the degree of white matter structure remaining within the infarct at three months may have implications for predicting patient outcome.
\end{abstract}

D iffusion tensor magnetic resonance imaging (DT-MRI) provides quantitative measures of the mobility of water molecules in vivo. Parameters such as the mean diffusivity $(<\mathrm{D}>)$, or the apparent diffusion coefficient (ADC) averaged over three orthogonal directions, measure the magnitude of diffusion of the water molecules, while diffusion anisotropy indices, such as fractional anisotropy (FA), indicate the deviation from pure isotropic diffusion of water mobility in vivo. These scalar parameters are thought to be useful markers of tissue cellular integrity.

Since the introduction of diffusion-weighted (DW)-MRI, many studies have investigated the temporal evolution of the parameters of diffusion in ischaemic stroke, most with DWMRI rather than with DT-MRI. ${ }^{1-9}$ Overall these studies found that the ADC decreases immediately after stroke, then increases slowly towards normal values (pseudonormalisation). Finally, it becomes elevated, which is thought to indicate tissue necrosis. Other authors report a general reduction in diffusion anisotropy after stroke, consistent with loss of integrity of the tissue structure. ${ }^{10}{ }^{11}$ These studies suggest that characterising the temporal evolution of the diffusion parameters may prove useful in differentiating acute from non-acute infarcts, as well as investigating how the brain structure is affected in ischaemic stroke.

There are, however, inconsistencies in the results of these studies. For example, some authors have reported a time of pseudonormalisation between five and 10 days, ${ }^{35}{ }^{8-11}$ while others found that the ADC reaches normal values between 24 and 48 hours $^{4}$ or at about one month. ${ }^{267}$ Generally, the diffusion anisotropy decreases after stroke, ${ }^{11}$ although some authors report an increase immediately after onset. ${ }^{10} 12$
Differences in imaging methodology may partly explain the conflicting results. ${ }^{13}$ Another possible cause could be heterogeneity of diffusion values within the lesion in the acute stage, potentially arising from the difference in the water diffusion properties of grey and white matter. ${ }^{14-16}$ Some investigators have considered this issue and measured the parameters of diffusion in ischaemic grey and white matter. ${ }^{10}{ }^{17-20}$ Unfortunately not all of these studies scanned individuals serially at each time point to control for between subject variability, ${ }^{17}{ }^{19}$ and most did not measure the full diffusion tensor. ${ }^{10} 1820$ Furthermore, some studies only imaged a small number of patients, ${ }^{18-20}$ while not all were specifically designed to analyse differences between the two types of tissue. ${ }^{10}{ }^{17}$ The purpose of the present study was therefore to investigate whether differences exist in the values and temporal evolution of $\langle\mathrm{D}>$ and FA of lesion grey and white matter in a group of patients imaged serially from onset to 3 months.

\section{SUBJECTS AND METHODS \\ Subjects}

Consecutive patients presenting with symptoms of moderate to severe cortical ischaemic stroke, who had no evidence of haemorrhage on the initial computed tomography (CT) or MR scan, were recruited during an effective period of 21 months between January 2001 and July 2003. A stroke

Abbreviations: $A D C$, apparent diffusion coefficient; $C T$, computed tomography; $\langle\mathrm{D}\rangle$, mean diffusivity; DT-MRI, diffusion tensor magnetic resonance imaging; DW-MRI, diffusion-weighted MRI; EP, echo planar; FA, fractional anisotropy; ROI, regions of interest 
physician examined all the patients prior to scanning, and categorised the type of stroke according to the Oxfordshire Community Stroke Project (OCSP) classification. ${ }^{21}$ The inclusion criteria for the study were as follows: (a) the patient could be imaged within a maximum of 24 hours after symptom onset, $(b)$ the patient had no contraindications to MR, and (c) the patient could tolerate the imaging protocol. For the present analysis, we identified those patients who were not receiving any thrombolytic treatment and whose visible ischaemic lesion on their first time point DW images included both grey and white matter. Patients underwent, where possible, repeat MRI at around 4-7 days, 10-14 days, 1 month, and 3 months after stroke. All patients were scanned at least twice. The local ethics committee approved the study, and in all cases informed consent was obtained from the patient or a close family member.

\section{Imaging methods}

All MRI data were obtained using a GE Signa LX $1.5 \mathrm{~T}$ (General Electric, Milwaukee, WI) clinical scanner, equipped with a self shielding gradient set $(22 \mathrm{mT} / \mathrm{m}$ maximum gradient strength and $120 \mathrm{~T} / \mathrm{m} / \mathrm{s}$ slew rate) and a "birdcage" quadrature head coil supplied by the manufacturer. The MRI examination consisted of a standard fast spin-echo T2weighted sequence, a gradient-echo Tl-weighted sequence, and a previously described DT-MRI protocol based on spinecho echo planar (EP) imaging. ${ }^{22}$ The duration of the examination was approximately 30 minutes.

To ensure that the slice locations used in the follow up scans corresponded as closely as possible to those in the first, the subject's head position and tilt in the first scan were recorded and the patient repositioned as close as possible to this location for the follow up scans. Computational image realignment techniques were then used to realign the images in the follow up scans to the first, thereby minimising any small remaining positioning errors and removing eddy current induced artefacts. ${ }^{23}$

From these MRI data, the apparent diffusion tensor of water (D) was calculated in each voxel from the signal intensities in the component EP images. ${ }^{24}$ Maps of $<\mathrm{D}>$ and FA for every patient at each time point were generated on a voxel-by-voxel basis from the sorted eigenvalues of $\mathbf{D}$ and converted into Analyze (Mayo Foundation, Rochester, MN) format.

\section{Region of interest analysis}

For each patient, the acute ischaemic lesion at less than 24 hours was outlined in each slice where the lesion was visible by tracing around the region of signal hyperintensity on the first DW-EP scan (fig 1A). The lesion outlines were copied onto the first scan T2-weighted EP images and multiple circular regions of interest (ROI) of $6 \mathrm{~mm}$ diameter were placed in grey and white matter within the lesion using anatomical and imaging knowledge (fig 1B). This ROI size was chosen to enable selection of regions of grey and white matter, while minimising the effects of mixed tissue and cerebrospinal fluid (CSF) contamination. ROI were also placed in comparable contralateral grey and white matter and were used as control regions for each patient. As many ROI as possible were placed in each slice of the T2-weighted EP image from the within 24 hours time point and then overlaid onto the coregistered DW-EP images, $\langle\mathrm{D}\rangle$, and FA parametric maps for the same time point (fig $1 \mathrm{C}, \mathrm{D}$ ) and all subsequent time points available for that patient. On average, for each patient 14 ROI were placed in grey matter and 10 in white matter. When tissue structures sampled by ROI chosen at the first time point were displaced in subsequent scans by lesion swelling or atrophy, the ROI positions were corrected using the structural $\mathrm{Tl}$ and $\mathrm{T} 2$-weighted imaging data so that

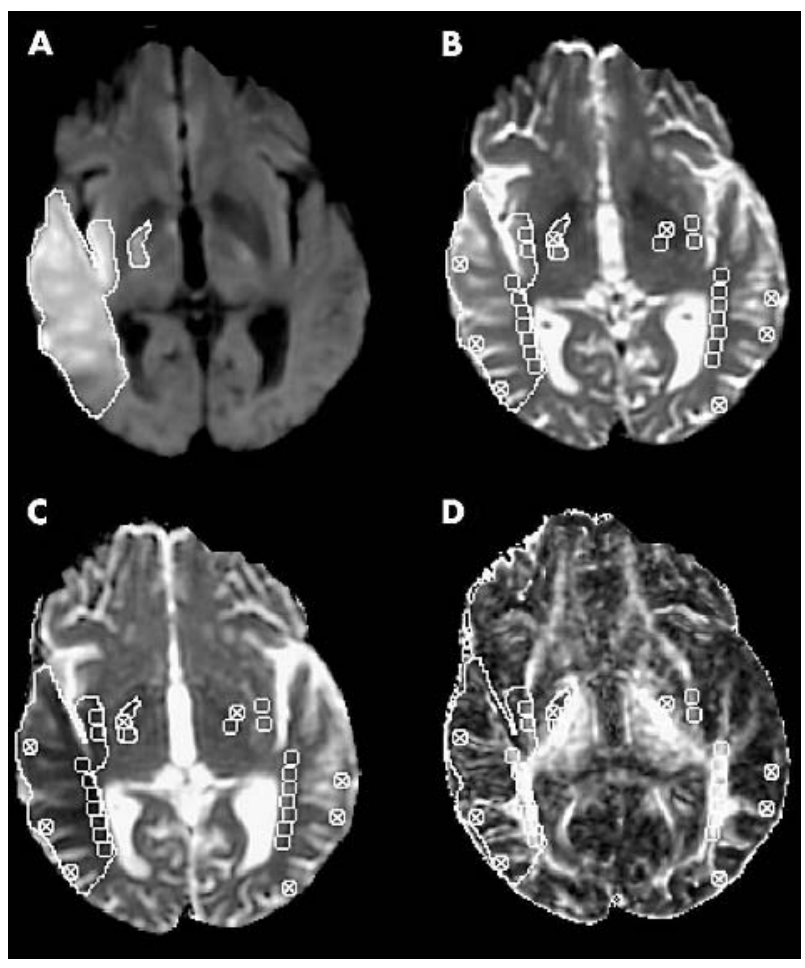

Figure 1 Examples of grey and white matter regions of interest (ROI) drawn on a representative slice from a 79 year old male patient with total right anterior circulation stroke. (A) Lesion outline drawn on the within 24 hours scan diffusion-weighted echo planar (DW-EP) image. (B) Circular ROI drawn in areas of grey and white matter in the lesion and in contralateral tissue using the coregistered T2-weighted EP images. All ROI copied onto (C) mean diffusivity $<D>$ and (D) fractional anisotropy (FA) maps for this time point and all subsequent scans to characterise the temporal evolution of these parameters. ROI with crosses indicate grey matter while open ROI indicate white matter.

the same anatomical piece of tissue was sampled at all time points. Areas of coincidental periventricular white matter disease and haemorrhagic transformation of the infarct at later time points were avoided with the aid of the structural MRI data. A neuroradiologist verified the positions of all ROI and the outlines of the ischaemic lesions.

\section{Statistical analysis}

Mean values of $<\mathrm{D}>\{$ grey $\},<\mathrm{D}>\{$ white $\}, F A\{$ grey $\}$ and FA $\{$ white $\}$ were calculated from all ROI in the lesion and contralateral tissue for every patient at each time point. However, while it is important to characterise the evolution of $\langle\mathrm{D}\rangle$ and FA to understand how stroke affects brain tissue structure, these absolute parameters also reflect the intrinsic difference between grey and white matter $(<\mathrm{D}>\{$ grey $\}>$ $<\mathrm{D}>\{$ white $\}$ and FA grey $\}<\mathrm{FA}\{$ white $\}$ for normal brain), and inter-patient variability. Therefore, to control for biological variation in $\angle \mathrm{D}\rangle$ and $\mathrm{FA}$ and to allow group comparisons between individuals at each time point, the ratio of lesion to contralateral $\angle \mathrm{D}\rangle$ and $\mathrm{FA}\left(\langle\mathrm{D}\rangle_{\mathrm{R}}\right.$ and $\left.\mathrm{FA}_{\mathrm{R}}\right)$ were also calculated. ${ }^{17-19}$ Finally, a global mean for each time point for grey and white matter $\langle\mathrm{D}\rangle, \mathrm{FA},\langle\mathrm{D}\rangle_{\mathrm{R}}$, and $\mathrm{FA}_{\mathrm{R}}$ was determined from the individual patient mean data. Thus, all lesions were weighted equally regardless of the number of ROI in each.

Differences between $\langle\mathrm{D}\rangle_{\mathrm{R}}$ and $\mathrm{FA}_{\mathrm{R}}$ values of grey and white matter at each time point for each individual patient were then assessed using two tailed Student's paired $t$ test (null hypothesis: $\quad<\mathrm{D}>_{\mathrm{R}}\{$ grey $\}-<\mathrm{D}>_{\mathrm{R}}\{$ white $\}=0$ and $\mathrm{FA}_{\mathrm{R}}\{$ grey $\}-\mathrm{FA}_{\mathrm{R}}\{$ white $\}=0$ ), with $\mathrm{p}<0.05$ considered as statistically significant. ${ }^{17-19}$ 


\section{RESULTS}

From a group of 109 patients recruited within 24 hours of stroke onset during the study period, 77 were excluded from the present analysis for the following reasons: (a) in 50 patients the lesion did not affect both grey and white matter, (b) 14 patients could not tolerate the entire first time point MRI examination, (c) in 10 patients primary haemorrhage was the cause of the stroke, $(d)$ data from two patients were corrupted, and $(e)$ one patient presented with evidence of other concurrent neurological disease on structural MRI. The remaining 32 patients, the majority with medium to large cortical infarcts involving both grey and white matter underwent DT-MRI examinations at least twice, with 18 patients scanned at all five time points. The demographics of the recruited patient group, stroke syndromes, and number of patients scanned at each time point are summarised in table 1 .

Figure 2 shows the variation in the absolute values of lesion $\angle \mathrm{D}>$ and FA after stroke for grey and white matter. After the initial reduction from normal values, both $<\mathrm{D}>\{$ grey $\}$ and $<\mathrm{D}>\{$ white $\}$ increased. The general trend in the evolution of $\langle\mathrm{D}\rangle$ grey $\}$ and $<\mathrm{D}>$ \{white $\}$ was similar, although between time points two (4-7 days) and three (10-14 days) the rate of increase of $<\mathrm{D}>$ \{grey was faster than $<\mathrm{D}>\{$ white $\}$. FA\{white $\}$ decreased rapidly during the first week, then more gradually over the next three weeks. FA \{grey\} decreased less rapidly than FA\{white during the first week, but then evolved similarly over the next three weeks. Between one and three months there was little change in either FA\{grey\} or FA\{white $\}$. However, although FA $\{$ white $\}$ decreased more than FA\{grey\} it was still higher at 3 months.

Values of $<\mathrm{D}>_{\mathrm{R}}\{$ grey $\}, \angle \mathrm{D}>_{\mathrm{R}}\{$ white $\}, \mathrm{FA}_{\mathrm{R}}\{$ grey $\}$, and $\mathrm{FA}_{\mathrm{R}}\{$ white $\}$ are shown in table 2 for all five time points. Both $<\mathrm{D}>_{\mathrm{R}}\{$ grey $\}$ and $<\mathrm{D}>_{\mathrm{R}}\{$ white $\}$ were significantly reduced during the first day after stroke onset $(\mathrm{p}<0.001)$. $<D>_{R}\{$ grey $\}$ was significantly higher than $<D>_{R}\{$ white $\}$ at this time point $(\mathrm{p}=0.02)$, with an average reduction of $26 \%$ for grey matter and $31 \%$ for white matter. Between the first and second time points $\langle\mathrm{D}\rangle_{\mathrm{R}}\{$ white $\}$ started to rise. $<\mathrm{D}\rangle_{\mathrm{R}}\{$ grey $\}$ and $\langle\mathrm{D}\rangle_{\mathrm{R}}\{$ white $\}$ were nearly identical at time point two. However, between time points two and three $<\mathrm{D}>_{\mathrm{R}}\{$ grey $\}$ increased at a faster rate and pseudonormalised earlier than $<\mathrm{D}>_{\mathrm{R}}\{$ white $\} .<\mathrm{D}>_{\mathrm{R}}\{$ grey $\}$ was again significantly higher than $\left\langle\mathrm{D}>_{\mathrm{R}}\{\right.$ white $\}$ at time point three $(p=0.03)$. This confirms the faster recovery of $<D>\{$ grey $\}$ seen between time points two and three in fig $2 \mathrm{~A}$. Thereafter, both $\angle \mathrm{D}\rangle_{\mathrm{R}}\{$ grey $\}$ and $\angle \mathrm{D}>_{\mathrm{R}}\{$ white $\}$ increased slowly over time becoming elevated and of the same value at 3 months. Table 2 also shows that while $\mathrm{FA}_{\mathrm{R}}$ \{grey\} remained unchanged, $\mathrm{FA}_{\mathrm{R}}\{$ white $\}$ was significantly reduced in the first day after stroke $(\mathrm{p}<0.001)$. Between time points one and two $\mathrm{FA}_{\mathrm{R}}\{$ grey $\}$ declined less rapidly than $\mathrm{FA}_{\mathrm{R}}\{$ white $\}$, with $\mathrm{FA}_{\mathrm{R}}\{$ grey $\}$ being reduced by $10 \%$ and $\mathrm{FA}_{\mathrm{R}}\{$ white $\}$ by $46 \%$. At
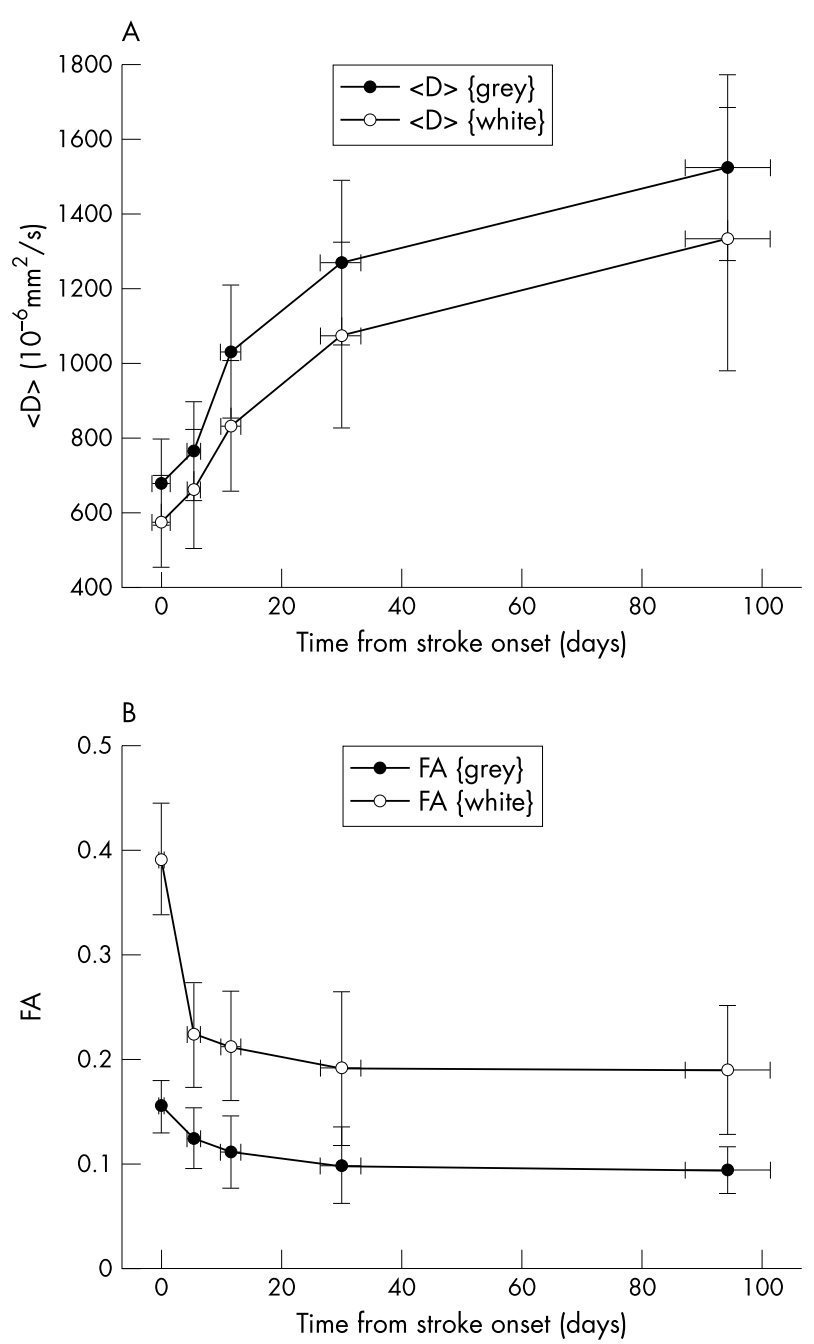

Figure 2 Temporal evolution of the absolute values of $(A)$ mean diffusivity $(\angle D>$ ) and (B) fractional anisotropy (FA) of lesion grey and white matter. For clarity, normal absolute values are not plotted. The mean (SD) absolute values for contralateral regions at all time points were $\angle D>=956(31) \times 10^{-6} \mathrm{~mm}^{2} / \mathrm{s}$ and $F A=0.14(0.01)$ for normal grey matter, and $\angle D>=831(17) \times 10^{-6} \mathrm{~mm}^{2} / \mathrm{s}$ and $\mathrm{FA}=0.43(0.02)$ for normal white matter.

later time points both $\mathrm{FA}_{\mathrm{R}}\{$ grey $\}$ and $\mathrm{FA}_{\mathrm{R}}\{$ white $\}$ continued to decrease gradually. $\mathrm{FA}_{\mathrm{R}}$ \{grey $\}$ was significantly greater than $\mathrm{FA}_{\mathrm{R}}\{$ white $\}$ at all time points $(\mathrm{p}<0.001)$.

\section{DISCUSSION}

This is the largest longitudinal study to date to investigate the temporal evolution of water diffusion parameters in grey and

Table 1 Demographic details, number of scans, and clinical stroke syndrome ${ }^{21}$ for the patients imaged in this study

\begin{tabular}{|c|c|c|c|c|c|c|c|}
\hline \multirow{2}{*}{$\begin{array}{l}\text { No. of } \\
\text { patients }\end{array}$} & \multirow{2}{*}{$\begin{array}{l}\text { No. of } \\
\text { scans }\end{array}$} & \multirow[b]{2}{*}{$M / F$} & \multirow{2}{*}{$\begin{array}{l}\text { Mean (SD) age } \\
\text { (years) }\end{array}$} & \multicolumn{4}{|c|}{ Stroke syndrome } \\
\hline & & & & TACS & PACS & LACS & POCS \\
\hline 18 & 5 & $12 / 6$ & $67.8(13.9)$ & 3 & 13 & 1 & 1 \\
\hline 6 & 4 & $4 / 2$ & $80.5(7.1)$ & 4 & 2 & - & - \\
\hline 5 & 3 & $2 / 3$ & $77.6(7.2)$ & 2 & 2 & - & 1 \\
\hline 3 & 2 & $2 / 1$ & $85.3(13.4)$ & 2 & - & - & 1 \\
\hline 32 & 135 & $20 / 12$ & 73.9 (13.3) & 11 & 17 & 1 & 3 \\
\hline
\end{tabular}

F, female; LACS, lacunar syndrome; M, male; PACS, partial anterior circulation syndrome; POCS, posterior circulation syndrome; TACS, total anterior circulation syndrome. 
Table 2 Mean (SD) ratios of ischaemic lesion to contralateral normal brain for mean diffusivity $\left(<D>_{R}\right)$ and fractional anisotropy $\left(F A_{R}\right)$ in grey and white matter at each time point

\begin{tabular}{|c|c|c|c|c|c|c|c|}
\hline \multicolumn{2}{|c|}{ Time (days) } & \multicolumn{3}{|l|}{$<\mathbf{D}>_{\mathrm{R}}$} & \multicolumn{3}{|l|}{$\mathrm{FA}_{\mathrm{R}}$} \\
\hline Window & Mean (SD) & Grey & White & $p$ & Grey & White & $p$ \\
\hline$<1$ & $0.3(0.3)$ & $0.74(0.13)$ & $0.69(0.14)$ & 0.02 & $1.00(0.19)$ & $0.86(0.13)$ & $<0.001$ \\
\hline $4-7$ & $5.5(1.1)$ & $0.79(0.13)$ & $0.79(0.18)$ & 0.99 & $0.90(0.17)$ & $0.54(0.14)$ & $<0.001$ \\
\hline $10-14$ & $11.7(1.7)$ & $1.05(0.19)$ & $0.97(0.19)$ & 0.03 & $0.80(0.22)$ & $0.52(0.15)$ & $<0.001$ \\
\hline 30 & 30.0 (3.4) & $1.32(0.23)$ & $1.28(0.29)$ & 0.47 & $0.73(0.26)$ & $0.46(0.17)$ & $<0.001$ \\
\hline 90 & $94.5(7.1)$ & $1.60(0.25)$ & $1.60(0.42)$ & 0.97 & $0.69(0.20)$ & $0.46(0.17)$ & $<0.001$ \\
\hline
\end{tabular}

Bold type indicates a significant difference $(p<0.05)$ between grey and white matter values at each time point. $\mathrm{SD}$, standard deviation.

white matter after stroke using DT-MRI. The above results show that there are differences in the temporal evolution of $<\mathrm{D}>$ and FA of grey and white matter in ischaemic lesions affecting both tissue types. Although the overall evolution of $<\mathrm{D}>\{$ grey $\}$ and $<\mathrm{D}>\{$ white $\}$ was similar, subtle differences were observed during the first two weeks after stroke, specifically the rate of increase of $\langle\mathrm{D}\rangle$ \{grey\} was faster between 4-7 and 10-14 days. Differences in the evolution of grey and white matter FA were more marked, with the loss of FA $\{$ white $\}$ being significantly greater than FA\{grey $\}$ during the first week after stroke.

Previous studies reporting changes in grey and white matter water diffusivity indices have shown contradictory results. As in the current study, Yang et al and Mukherjee et al have found that $\langle\mathrm{D}\rangle_{\mathrm{R}}$ was significantly more reduced in white than grey matter at less than three days. ${ }^{10}{ }^{19}$ However, Sorensen et al reported that $\langle\mathrm{D}\rangle_{\mathrm{R}}$ was significantly more reduced in grey matter, ${ }^{17}$ while Bastin et al and Fiebach et al did not find significant differences between grey and white matter at this stage. ${ }^{18} 20$ In the longitudinal studies of Yang et al and Fiebach et al, it was observed that $\langle\mathrm{D}\rangle_{\mathrm{R}}$ of both grey and white matter remained low during approximately the first four days, increasing thereafter, and reaching normal contralateral values at approximately day $10 .{ }^{10}{ }^{20}$ Conversely, Bastin et al only found this pattern of $\langle\mathrm{D}\rangle_{\mathrm{R}}$ change in grey matter, which started to rise at approximately seven days, while no significant change was observed in white matter water $<\mathrm{D}\rangle_{\mathrm{R}}$ during the first 14 days after the initial reduction following stroke. ${ }^{18}$ However, all these studies, as with the current work, observed a slightly earlier renormalisation of grey matter water diffusivity.

Our values of $\mathrm{FA}_{\mathrm{R}}$ at less than 24 hours are similar to the results of Sorensen et $a l^{17}$ who found that grey matter FA remained unchanged at this time point while white matter FA decreased significantly, even though only 10 of the 50 patients studied had lesions affecting both grey and white matter. Yang et al reported changes in both grey and white matter diffusion anisotropy. ${ }^{10}$ In their longitudinal study of 26 patients, most subjects showed an increase of diffusion anisotropy for both tissue types by 12 hours, after which the diffusion anisotropy declined. At approximately 90 days, the diffusion anisotropy was further reduced in all lesions. However, for most patients in their study the diffusion anisotropy was assessed by means of an orientation dependent parameter derived from ADC values, rather than a rotationally invariant index derived from $\mathbf{D}$, such as FA.

Elevated $\langle\mathrm{D}\rangle$ and reduced $\mathrm{FA}$ indicate destruction of membrane integrity and progression towards necrosis. ${ }^{25}$ Interestingly, fig $2 \mathrm{~A}$ shows that grey and white matter $<\mathrm{D}>$ was still increasing at three months, while FA did not change significantly after one month (fig 1B). Increasing water diffusion at later time points has been observed in other studies, which have attributed this to gliosis and accumulation of CSF in the lesion secondary to encephalomalacia ${ }^{1}$ or to flow dephasing effects in regions that have undergone cystic degeneration. ${ }^{5}$ However, no measurements of diffusion anisotropy were made in these studies to compare with ours. Another important observation is that despite the greater loss of white matter FA, it was still significantly higher than grey matter FA at three months, indicating that some organised axonal structure remains. This effect was more marked in some patients than others, which contributes to the larger standard deviations measured in FA\{white $\}$ than FA\{grey\} (fig 2B). To our knowledge, this has not been reported before, as such effects are not evident when measuring whole lesion diffusion parameters, and could have implications in predicting patient outcome. For instance, DT-MRI tractography methods could be used to measure how changes in tract volume and integrity following stroke relate to clinical dysfunction and outcome.

\section{CONCLUSIONS}

By following individual patients over time with ischaemic lesions affecting both grey and white matter, we found evidence for the difference in the values and temporal evolution of $\langle\mathrm{D}>$ and FA for grey and white matter. Specifically, the rate of increase of $\langle\mathrm{D}>$ \{grey $\}$ was faster than $\langle\mathrm{D}>\{$ white $\}$ from week one to week two, while FA\{white $\}$ decreased more rapidly than FA\{grey\} during the first week after stroke. Thereafter, FA decreased gradually for both tissue types. However, FA\{white $\}$ was still higher than FA\{grey\} at three months indicating that some organised axonal structure remained. The observation that there is patient-to-patient variability in the degree of white matter structure remaining within the infarct at three months may have implications for predicting patients' outcome.

\section{Authors' affiliations}

S Muñoz Maniega, M E Bastin, Medical and Radiological Sciences (Medical Physics), University of Edinburgh, Edinburgh, UK

P A Armitage, A J Farrall, TK Carpenter, P J Hand, V Cvoro, C S Rivers, J M Wardlaw, Clinical Neurosciences, University of Edinburgh, Edinburgh, UK

This work was funded by the Chief Scientist Office of Scottish Office, the Stroke Association, the Row Fogo Charitable Trust and the Medical Research Council, and was undertaken at the SHEFC Brain Imaging Research Centre for Scotland (http://www.den.ed.ac.uk/bic).

Competing interests: none declared

\section{REFERENCES}

1 Chien D, Kwong KK, Gress DR, et al. MR diffusion imaging of cerebral infarction in humans. AJNR Am J Neuroradiol 1992;13:1097-102.

2 Warach S, Chien D, Li W, et al. Fast magnetic resonance diffusion-weighted imaging of acute human stroke. Neurology 1992;42:1717-23. 
3 Warach S, Gaa J, Siewert B, et al. Acute human stroke studied by whole brain echo planar diffusion-weighted magnetic resonance imaging. Ann Neurol 1995;37:231-41.

4 Welch KMA, Windham J, Knight RA, et al. A model to predict the histopathology of human stroke using diffusion and T2-weighted magneticresonance-imaging. Stroke 1995;26:1983-9.

5 Marks MP, deCrespigny A, Lentz D, et al. Acute and chronic stroke: navigated spin-echo diffusion-weighted MR imaging. Radiology 1996;199:403-8.

6 Lutsep HL, Albers GW, deCrespigny A, et al. Clinical utility of diffusionweighted magnetic resonance imaging in the assessment of ischemic stroke. Ann Neurol 1997;41:574-80.

7 Schwamm LH, Koroshetz WJ, Sorensen AG, et al. Time course of lesion development in patients with acute stroke-serial diffusion- and hemodynamic-weighted magnetic resonance imaging. Stroke 1998;29:2268-76.

8 Schlaug G, Siewert B, Benfield A, et al. Time course of the apparent diffusion coefficient (ADC) abnormality in human stroke. Neurology 1997;49:113-19.

9 Ahlhelm F, Schneider G, Backens M, et al. Time course of the apparent diffusion coefficient after cerebral infarction. Eur Radiol 2002;12:2322-9.

10 Yang Q, Tress BM, Barber PA, et al. Serial study of apparent diffusion coefficient and anisotropy in patients with acute stroke. Stroke 1999;30:2382-90.

11 Zelaya F, Flood N, Chalk JB, et al. An evaluation of the time dependence of the anisotropy of the water diffusion tensor in acute human ischemia. Magn Reson Imaging 1999;17:331-48.

12 Armitage PA, Bastin ME, Marshall I, et al. Diffusion anisotropy measurements in ischaemic stroke of the human brain. MAGMA 1998:6:28-36.

13 Uluğ AM, Beauchamp N, Bryan RN, et al. Absolute quantitation of diffusion constants in human stroke. Stroke 1997;28:483-90.
14 Kuroiwa $T$, Nagaoka T, Ueki $M$, et al. Different apparent diffusion coefficient-water content correlations of gray and white matter during early ischemia. Stroke 1998;29:859-65.

15 Nagesh V, Welch KMA, Windham JP, et al. Time course of $A D C(w)$ changes in ischemic stroke: beyond the human eye! Stroke 1998;29:1778-82.

16 Mukherjee P, McKinstry RC, Shimony JS, et al. Heterogeneity of apparent diffusion coefficients within infarcts. Stroke 2000;32:1695-6.

17 Sorensen AG, Wu O, Copen WA, et al. Human acute cerebral ischemia: detection of changes in water diffusion anisotropy by using MR imaging. Radiology 1999:212:785-92.

18 Bastin ME, Rana AK, Wardlaw JM, et al. A study of the apparent diffusion coefficient of grey and white matter in human ischaemic stroke. NeuroReport 2000;11:2867-74.

19 Mukherjee $\mathrm{P}$, Bahn MM, McKinstry RC, et al. Differences between gray matter and white matter water diffusion in stroke: diffusion-tensor MR imaging in 12 patients. Radiology 2000;215:211-20.

20 Fiebach JB, Jansen O, Schellinger PD, et al. Serial analysis of the apparent diffusion coefficient time course in human stroke. Neuroradiology 2002;44:294-8.

21 Bamford JM, Sandercock PAG, Dennis M, et al. Classification and natural history of clinically identifiable subtypes of cerebral infarction. Lancet 1991;337:1521-6

22 Sinha S, Bastin ME, Whittle IR, et al. Diffusion tensor MR imaging of highgrade cerebral gliomas. AJNR Am J Neuroradiol 2002;23:520-7.

23 Jenkinson M, Smith S. A global optimisation method for robust affine registration of brain images. Med Image Anal 2001;5:143-56.

24 Basser PJ, Mattiello J, LeBihan D. Estimation of the effective self-diffusion tensor from the NMR spin echo. J Magn Reson 1994;103:247-54

25 Pierpaoli C, Righini A, Linfante I, et al. Histopathologic correlates of abnormal water diffusion in cerebral ischemia: diffusion-weighted MR-imaging and light and electron-microscopic study. Radiology 1993;189:439-48. 